\section{A lethal injection}

The stealthy assassin creeps up to the victim and quickly injects a dose of deadly poison. No, it's not a scene from a spy novel, but one of the ways in which the immune system's natural assassins - cytotoxic T lymphocytes and natural killer cells - can kill their target cells. The deadly poison in this case is a serine protease called granzyme B (GrB), and the killing mechanism is discussed by Christopher Froelich and colleagues in The Journal of Cell Biology.

Much is known about GrB - there's evidence that it kills with or without caspase activation, and that it causes mitochondrial permeabilization with or without members of the Bcl-2 family. So Froelich and co-workers set out to assess the relative contributions of these pathways, to come up with a probable scheme for how GrB normally acts. To do this, they measured several different hallmarks of apoptosis - loss of mitochondrial membrane potential $\left(\psi_{\mathrm{m}}\right)$, DNA fragmentation and activation of procaspase precursors.

Most previous studies have been done on cell lysates, after GrB has been delivered to its target. By contrast, Froelich and colleagues studied whole cells after delivery of the physiological form of GrB, a GrB-Serglycin (SG) complex. Previous studies with lysates had indicated that GrB-treated cells rapidly acquire cleaved (and presumably active) procaspase- 3 . Mature caspase- 3 and GrB then process caspase- 7 to complete the initial phase of apoptosis. The authors therefore asked how the absence of procaspase-3 might alter the pattern of apoptosis in whole cells after delivery of GrB-SG.

The authors compared MCF-7 cells that lacked procaspase-3 $\left(\mathrm{MCF}^{-}{ }_{\mathrm{vec}}\right)$ with cells that contained a stable transfectant that expressed this zymogen (MCF-7 ${ }_{\text {casp-3 } 3}$ ), after delivery of GrB-SG. They saw DNA fragmentation and a loss of $\psi_{\mathrm{m}}$ in the MCF-7 ${ }_{\text {casp- } 3}$ cells, but not in MCF-7 $7_{\text {vec }}$ cells, indicating that caspase- 3 is essential for starting apoptosis. The authors also showed that the requirement for caspase- 3 was not due simply to the fact that caspase- 7 could no longer be activated in the caspase-3-deficient cells.

If caspase- 3 is the crucial initiator, what's the contribution of the Bcl-2-family members? First, the pro-apoptotic members - such as Bax, Bak and the socalled 'BH3-only' protein BID - might be involved in mitochondrial permeabilization by forming large ion channels in the outer mitochondrial membrane. Froelich and colleagues looked into this by studying the sensitivity of $\mathrm{Bax} / \mathrm{Bak}(-/-)$ mouse embryo fibroblasts (MEFs) to GrB-mediated killing. The Bax/Bak (-/-) MEFs showed lower levels of active caspase-3 intracellularly and lower rates of $\psi_{\mathrm{m}}$ loss than wild-type MEFs, which indicates that these proteins are required for the response to GrB. The authors also studied whether BID is cleaved in response to GrB. Unlike previous studies, however, they could not detect the truncated (active) form of BID when precautions were taken to minimize proteolysis during processing of cells for immunoblots.

A second role for certain members of the Bcl-2 family is in preventing cell death. The eponymous Bcl-2 has been shown to block GrB-mediated apoptosis, and the authors wondered whether it might do this by acting directly on caspase- 3 . They therefore looked at the effects of GrB-SG in a Jurkat cell line that overexpressed Bcl-2. In contrast to wild-type cells, the Jurkat ${ }_{\mathrm{Bc}-2}$ cells showed no caspase-3 activity, loss of $\psi_{\mathrm{m}}$ or DNA fragmentation. Bcl-2 did not inhibit isolated active caspase- 3 , and processed caspase- 3 was observed by immunoblot, leading the authors to conclude that Bcl-2 might suppress apoptosis by blocking the assembly of processed caspase- 3 .

On the basis of these and several other observations, Froelich and colleagues suggest that the predominant GrB death pathway starts with the activation of procaspase- 3 , and that the death signal is maximized by mitochondria through caspase-mediated engagement of the $\mathrm{BH} 3-o n l y / \mathrm{Bax} / \mathrm{Bak}$ pathway. The $\mathrm{BH}$ 3-only protein that's cleaved by caspase- 3 might be BID, but Froelich and colleagues' results indicate that there might be other participants too.

\section{(2) References and links}

Alison Mitchell

ORIGINAL RESEARCH PAPER Metkar, S. S. et al. Granzyme B activates procaspase-3 which signals a mitochondrial amplification loop for maximal apoptosis. J. Cell Biol. 160, 875-885 (2003)

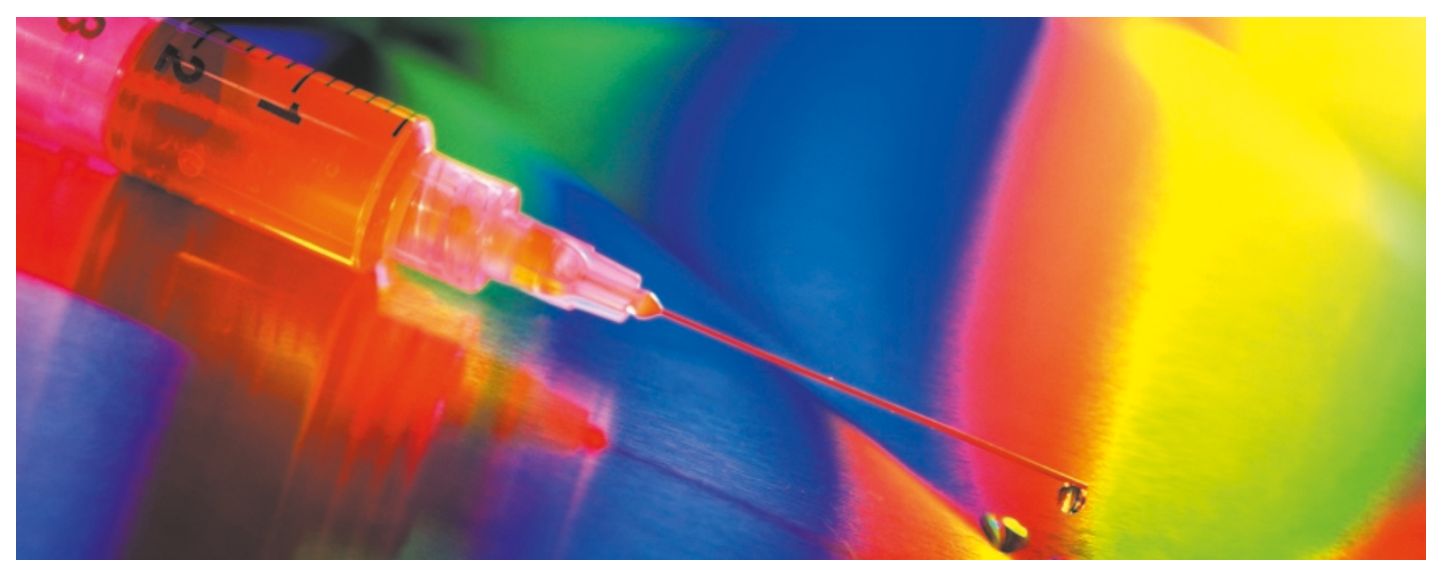

\section{IN THE NEWS}

Caught in the act

Scientists believe that prion

diseases - such as scrapie

in sheep, 'mad-cow

disease' in cattle and

Creutzfeldt-Jakob disease

in humans - are promoted

by the conversion of norma cellular prion protein $\left(\mathrm{PrP}^{\mathrm{C}}\right)$

into the disease-inducing

form $\left(\mathrm{PrP}^{\mathrm{Sc}}\right)$. A new study in Cell now shows that the two types of prions bind to each other, and that an engineered prion form might help cure disease. Technical obstacles had previously prevented the demonstration of a physical interaction between $\mathrm{PrP}^{\mathrm{C}}$

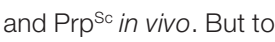
overcome certain practical impediments, Adriano Aguzzi, from the University Hospital of Zürich,

Switzerland, and his colleagues engineered an artificial prion - PrP- $\mathrm{FC}_{2}$ that consisted of soluble, dimerized $\mathrm{PrPC}^{\mathrm{C}}$ fused to immunoglobulin Fc $\gamma$.

When the soluble dimeric prion protein was expressed in transgenic mice, which were subsequently inoculated with scrapie prion, Aguzzi and colleagues found that PrPsc accumulation, prion replication and onset of disease were significantly delayed.

The researchers could also show - using immunoprecipitation and pull-down asays - that $\mathrm{PrP}-\mathrm{FC}_{2}$ and PrPSc interacted, in vitro and in vivo. "It's the cleanest evidence yet" for the interaction, says prion researcher Michael Scott of the University of California in San Francisco. "It mimics the normal situation."

(Nature Science Update, $4^{\text {th }}$ April 2003).

"PrPFc ${ }_{2}$ is capable of interfering with the replication of pathological prion and the effect is very robust...", says Aguzzi (Cell Press Release, $3^{\text {rd }}$ April 2003). This suggests that soluble PrP derivatives might represent a new class of antiprion agents.

Arianne Heinrichs 